\title{
Introduction : La fibrose cicatricielle radioinduite
}

\author{
M. MARTIN, J.-L. LEFAIX \\ Laboratoire de radiobiologie appliquée, Commissariat à l'énergie atomique, \\ DSV/DPTE, 91191 Gif-sur-Yvette Cedex
}

Chez les mammifères adultes, les cellules conjonctives sont en majorité dans un état de quiescence, caractérisé par une différenciation stable et une absence de division cellulaire. A la suite d'une agression tissulaire, ces cellules peuvent être transitoirement activées dans le but de réparer les lésions provoquées. Dans certaines pathologies, une altération de ces processus conduit à une activation chronique des populations fibroblastiques. C'est dans ce type de lésions que peuvent se classer les fibroses pathologiques et la fibrose radioinduite en particulier.

Les mécanismes sous-jacents à la formation de la fibrose et aux activations cellulaires ont été beaucoup étudiés, mais ils restent encore mal compris. Par ailleurs, aucun traitement classique n'a fait preuve d'une réelle efficacité, en préventif comme en curatif.

Nous définirons la fibrose radioinduite dans ses diverses formes cliniques et par sa composition. Ceci permettra d'introduire les données nouvelles présentées pendant cette journée d'étude.

\section{Définition de la fibrose.}

La fibrose radioinduite est une fibrose cicatricielle qui est observée après une surexposition à des radiations ionisantes. Etymologiquement, la fibrose est une hyperplasie conjonctive avec prolifération de fibroblastes et accumulation de fibres de collagène. Elle est susceptible de siéger au niveau de tous les organes. Malgré cette grande diversité de localisation, la fibrose présente des caractéristiques remarquablement conservées, car elle est toujours la conséquence d'une rupture de deux équilibres: celui qui règle la synthèse et la dégradation de la matrice extracellulaire et celui qui règle l'état quiescent ou proliférant des cellules mésenchymateuses. 


\section{Origine de la fibrose}

\subsection{Radiothérapie et fibrose}

La fibrose constitue une des complications majeures de la radiothérapie. Elle peut être observée dans presque tous les tissus et organes après irradiation fractionnée et évolue sur des périodes très longues, de l'ordre de plusieurs années. Son importance varie en fonction du type de traitement, du type de tumeur et du terrain individuel [2, 10-12].

La fibrose intervient plus fréquemment lorsque la dose d'irradiation est élevée, lorsqu'il existe des recoupes dans les champs d'irradiation ou que l'appareil utilisé présente un mauvais rendement en profondeur. Elle est d'autant plus grave qu'elle concerne un organe vital (entérite, péricardite) ou des extrémités (plexite radique, déformation faciale). Le tissu cicatriciel reste fragile et peut être le siège de réactivations inflammatoires à la suite de traumatismes mineurs ou d'infections.

Dans le cas du cancer du sein, les anciens procédés d'irradiation provoquaient souvent l'induration complète du tissu mammaire. Les procédés plus récents ont permis de limiter la dose à la peau et de diminuer le volume de tissu fibreux.

Bien que les méthodes modernes de radiothérapie en mégavoltage permettent de réduire notablement la fréquence des fibroses radioinduites, les radiothérapeutes expriment le besoin de molécules nouvelles capables de prévenir ou de réduire ces complications tardives. De plus, l'introduction de nouveaux types de rayonnements dans les protocoles thérapeutiques, tels que les neutrons et les ions lourds, rend nécessaire l'étude des complications tardives induites par ces rayonnements.

\subsection{Accidents d'irradiation et fibrose}

En France, les cas d'accidents d'irradiation sont observés essentiellement en milieu industriel. En effet, sur plus de deux cents accidents pendant les 25 dernières années, $85 \%$ sont survenus en milieu industriel et $15 \%$ en milieu médical. Ce sont essentiellement des irradiations localisées à fortes doses, concernant les mains, les cuisses, le thorax et les pieds, par ordre de fréquence décroissante. Ce type d'accident s'est multiplié avec le développement des techniques de gammagraphie industrielle qui permettent un contrôle non destructif des pièces métalliques à l'aide d'appareils portables munis de sources d'iridium 192. Des accidents d'irradiation par rayons $X$ ont été relatés également en cristallographie et en analyse par fluorescence $X[4,9]$.

Les lésions occasionnées sont souvent graves, allant de l'érythème pour une dose comprise entre 3 et $8 \mathrm{~Gy}$, à la nécrose puis à la fibrose au-delà de 25 Gy [5]. Le traitement se limite actuellement à la chirurgie en raison de l'absence de thérapeutique adaptée. Les cliniciens ont souvent observé un phénomène d'évolution tardive en cycles pour ces 
lésions: après l'obtention d'une guérison qui semble définitive, une réactivation des lésions peut s'observer, avec de nouvelles poussées inflammatoires, nécessitant une réintervention chirurgicale. L'origine de ces phases de réactivation demeure totalement inconnue.

\section{Formation de la fibrose}

Les effets biologiques d'une irradiation résultent d'une chaîne de réactions physico-chimiques qui est déclenchée par le passage du rayonnement. Les événements initiaux sont les ionisations et les excitations des atomes le long des trajectoires des particules ionisantes. Des radicaux libres se forment, notamment par radiolyse de l'eau, et provoquent des altérations moléculaires et des lésions cellulaires. Celles-ci se traduisent au niveau des tissus par des effets immédiats et tardifs.

Concernant les effets tardifs, la pathologie de l'irradiation aiguë peut se résumer par la nécrose ou l'atrophie du parenchyme, suivie par le développement d'une fibrose [2]. L'atrophie du parenchyme de l'organe irradié survient à la suite de la mort cellulaire immédiate ou différée, en particulier dans les cellules endothéliales de l'arbre vasculaire. Ces atteintes se manifestent précocément par une augmentation de la perméabilité de l'endothélium, une extravasation des protéines plasmatiques et une réaction inflammatoire locale. Ces zones d'oedème sont des zones d'attraction pour les fibroblastes, qui vont synthétiser les éléments de la fibrose.

\section{Composition de la fibrose}

\subsection{La matrice extracellulaire}

Le tissu fibreux cicatriciel est composé des quatre grandes familles de molécules du tissu conjonctif : les collagènes, les glycoprotéines, l'élastine et les protéoglycanes.

Le collagène est le composant macromoléculaire majeur de la matrice extracellulaire. C'est également le composant principal des tissus fibreux cicatriciels. Le gène ancestral du collagène a été dupliqué un grand nombre de fois, ce qui a généré la famille complexe des protéines collagéniques. Dans la fibrose, les isotypes de collagène les mieux décrits actuellement sont les types I, III, IV, V et VI. Un des traits principaux de la fibrose est une déposition excessive des collagènes intersticiels de type I et III [14].

La fibronectine est la principale glycoprotéine des tissus cicatriciels. D'une manière générale, la fibronectine joue un rôle important dans les phases précoces de la formation du tissu conjonctif, en particulier pendant le développement embryonnaire et la cicatrisation. 
Les glycosaminoglycanes sont des polysaccharides composés d'unités répétitives en nombre variable. Dans les tissus, ils sont sous forme de protéoglycanes, macromolécules complexes qui contiennent un noyau protéique lié à un nombre variable de chaînes de glycosaminoglycanes. Des particularités ont été décrites dans la fibrose pour ces composés, soit quantitative, comme une accumulation d'acide hyaluronique, soit qualitative, comme une sous-sulfatation.

\subsection{Les composants cellulaires}

Le fibroblaste, cellule d'origine mésodermique, est la cellule clé du tissu conjonctif, car il assure l'équilibre dynamique entre la synthèse et la dégradation de la matrice extracellulaire. II est avant tout une cellule sécrétrice qui élabore les différents constituants du tissu conjonctif. II est également capable de sécréter un grand nombre de protéinases exercant un rôle déterminant sur la dégradation et le renouvellement de la matrice extracellulaire. Enfin, les facteurs de croissance qu'il est capable de sécréter, comme l'interféron-b, le PDGF, l'EGF, le TGF-B ou les interleukines, en font également une cellule régulatrice.

Dans le tissu conjonctif mature, le fibroblaste est une cellule quiescente et fusiforme. Sous l'effet de divers stimuli, le fibroblaste peut devenir hyperactif. Ainsi, au cours des réactions inflammatoires et de la cicatrisation, Gabbiani [3] a décrit l'existence d'une cellule particulière, le myofibroblaste, doué de propriétés contractiles, sécrétoires et macrophagiques. Ce type cellulaire est retrouvé dans la fibrose et il y joue un rôle central [7].

Pendant la phase précoce d'évolution de la lésion, la cellule endothéliale joue un rôle important, toujours lié à sa dégénérescence. En effet, l'inflammation commence généralement par un défaut de la paroi vasculaire, qui permet l'extravasation des protéines sériques et la formation de l'oedème. Dans une seconde phase, la destruction des cellules endothéliales conduit à la nécrose des vaisseaux sanguins et à une ischémie locale, qui joue un rôle fondamental dans le développement de la fibrose. La cellule endothéliale reste souvent présente dans le tissu fibreux à la suite des phénomènes de néovascularisation, qui sont généralement associés à une extension de la fibrose plutôt qu'à sa limitation [12].

Les études histologiques ont décrit l'association fréquente entre les cellules de la réaction inflammatoire et les fibroblastes dans les tissus fibreux cicatriciels. Les types cellulaires impliqués sont variables suivant le siège anatomique, le type de fibrose et la phase considérée. Lors de la découverte des cytokines, lymphokines et facteurs de croissance, de nombreux auteurs ont formulé l'hypothèse selon laquelle les cellules mononucléées produiraient des facteurs solubles spécialisés qui induisent et perpétuent la réponse fibroplasique. 


\section{Problématique de la fibrose}

Malgré la diversité des facteurs étiologiques et des localisations, toutes les fibroses radioinduites présentent un certain nombre de traits communs, dont les principaux sont une destruction progressive du parenchyme, une infiltration inflammatoire, une prolifération des fibroblastes et une accumulation de matrice extracellulaire. Les recherches actuelles s'orientent autour de la question centrale des mécanismes de ces activations cellulaires, tant au stade de l'initiation des lésions que pendant la phase chronique de fibrose.

Lors d'une cicatrisation normale, les processus d'inflammation cessent dès que le comblement est assuré. D'une manière encore inconnue, l'organisme envoie des signaux de rétrocontrôle qui provoquent la disparition des cellules de l'inflammation et le retour des fibroblastes à un état quiescent. Inversement, dans les pathologies fibreuses, ce rétro-contrôle n'existe pas et les lésions deviennent alors chroniques, comme si l'agent causal, l'agresseur cellulaire, était toujours présent. L'infiltration par les cellules de l'inflammation est alors permanente et les fibroblastes restent dans un état activé.

Au cours de la phase d'initiation des lésions, parmi les nombreux messagers intercellulaires récemment découverts, certains facteurs de l'inflammation comme le TNF (tumor necrosis factor) et les interleukines pourraient être impliqués dans le développement de la fibrose. Leur origine cellulaire, leur cible ainsi que leur véritable rôle in vivo restent à déterminer.

Lors du stade de fibrose chronique, les différents types cellulaires en présence ainsi que les produits de dégradation tissulaires et cellulaires ont des rôles qui restent à définir. Des facteurs de croissance comme le TGF- $\beta$ pourraient jouer un rôle dans l'activation chronique des fibroblastes. En effet, le TGF-ß peut à la fois activer la synthèse de la matrice extracellulaire et diminuer la synthèse et l'activation des enzymes protéolytiques qui dégradent cette matrice [8].

Le rôle des radicaux libres oxygénés est encore débattu. Ils peuvent intervenir dans la formation de la fibrose selon deux mécanismes. En effet, des lésions de l'ADN sont provoquées par les radicaux libres formés lors du passage initial des rayonnements ionisants. Les lésions non réparées peuvent conduire à des anomalies chromosomiques transmissibles et à des mutations. Mais les dérivés de l'oxygène moléculaire sont également produits en excès dans les états inflammatoires aigus et chroniques. Ces radicaux libres pourraient participer au développement d'une fibrose dans un contexte d'inflammation chronique et d'ischémie tissulaire.

\section{Perspectives thérapeutiques}

Les potentialités anti-fibroplasiques de nombreuses molécules ont été testées dans des cultures de cellules et sur des modèles expérimentaux 
$[6,13]$. Un certain nombre de ces composés agissent au niveau de la régulation post-traductionnelle du collagène et se révèlent capables d'inhiber son accumulation. Ainsi le beta-aminopropionotrile et la D-pénicillamine sont-ils actifs au niveau de la polymérisation des fibres de collagène. Mais l'utilisation en clinique de ces composés est limitée par leur toxicité. Par ailleurs, des essais menés avec la colchicine chez des patients présentant une fibrose hépatique se sont révélés encourageants. Par leurs propriétés anti-inflammatoires, les corticoïdes restent le traitement de référence des réactions fibreuses, mais ils demeurent d'une efficacité limitée.

Des travaux récents ouvrent de nouvelles perspectives thérapeutiques. Ainsi, des traitements visant à réduire la réaction fibroplasique peuvent être envisagés àvec des molécules comme l'interféron gamma ou la superoxyde dismutase.

Aujourd'hui, les techniques modernes de la biologie cellulaire et moléculaire donnent la possibilité de progresser rapidement dans la compréhension des mécanismes de formation de ces pathologies. C'est pourquoi les recherches sur ce sujet se développent tant au niveau clinique que biologique et donnent lieu à des échanges scientifiques, tels cette table ronde sur la fibrose radique.

\section{RÉFÉRENCES}

[1] Emami B., Lyman J., Coia L., Goitein M., Munenrider J. E., Shank B., Solin L. J., and Wesson M. - Tolerance of normal tissue to therapeutic irradiation. Int. J. Radiat. Oncol. Biol. Phys., 1991, 21, 109-122.

[2] Fajardo L. F. - Pathology of radiation injury. New-York : Masson, 1982.

[3] Gabbiani G. - The myofibroblast: a key cell for wound healing and fibrocontractive diseases. In : Connective tissue research : chemistry, biology and physiology. New York : A.R. Liss, 1981, 183-194.

[4] Galle P., Masse R., Nenot J.C., Eds - Actualités sur les irradiations accidentelles et thérapeutiques, Créteil, 6-8 juin 1984. Paris : Masson, 1984.

[5] Jammet $H$. et Gongora R. - Radiolésions aiguës localisées: intérêt de la dosimétrie physique et biologique; étude clinique. In: II systema tegumentario e le radiazioni ionizzanti, St Vincent, 13-15 Sept 1981 (Strambi E., Eds). Rome : Associazione italiana radioprotezione medica, 1981, vol. 1, 9-39.

[6] Kivirikko K., Majmaa K. - Synthesis of collagen: chemical regulation of post-translational events. In : Fibrosis. (Ciba foundation symposium, 114). London : Pitman, 1985, 34-64.

[7] Martin M., Lafuma C., Sabatier L., Crechet F., Pinton P., Daburon F. Deterministic late effects of skin irradiation: in vitro studies of experimental radiation fibrosis. In : Skin dosimetry - radiological protection, aspects of skin irradiation, Dublin, 13-15 mars 1991 (Menzel H.G., Ed.). Radiat. Prot. Dos., 1991, 39 (1/3) 7-10.

[8] Roberts A. B. - The transforming growth factor-Bs. In : Peptide growth factors and their receptors (M. B. Sporn, A. B. Roberts, Eds.). Berlin : Springer-Verlag, 1990, vol. 1, pp 419-472. 
[9] Rodrigues de Oliveira A. - Un répertoire des accidents radiologiques 1945-1985. Radioprotection, 1987, 22 (2) 89-135.

[10] Réunion sur les effets des radiations ionisantes, Créteil, mai 1989. J. Med. Nucl. Biophys., 1990, 14 (2) 161-187.

[11] Radiobiologie des complications tardives, Congrès National de la SFRO, Paris, 22-23 novembre 1990. Bull. Cancer/ Radiother., 1990, 77 (4) 323-327.

[12] Thames H. D., Bentzen S. M., Turesson I., Overgaard M., Van den Bogaert W. Time-dose factors in radiotherapy: a review of the human data. Radiother. Oncol., $1990,19,219-235$.

[13] Voisin C. - Treatment of diffuse interstitial fibrosis. In : Tissue fibrosis: immune cells and mediators (J. P. Revillard and N. Wierzbicki, Eds). (Fondation francoallemande, international symposium series, 3), 1987.

[14] Wegrowski J., Lafuma C., Lefaix J-L., Daburon F., Robert L. - Modification of collagen and non collagenous proteins in radiation-induced muscular fibrosis. Exp. Mol. Pathol., 1988, 48, 273-285. 